International Journal of Pure and Applied Mathematics

Volume 89 No. 2 2013, 153-172

ISSN: 1311-8080 (printed version); ISSN: 1314-3395 (on-line version)

url: http://www.ijpam.eu

doi: http://dx.doi.org/10.12732/ijpam.v89i2.4

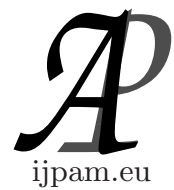

\title{
THE ROTA METHOD FOR SOLVING POLYNOMIAL EQUATIONS: A MODERN APPLICATION OF INVARIANT THEORY
}

\author{
Kris Jorgenson \\ Sul Ross State University \\ Alpine, TX 79830, USA
}

\begin{abstract}
Algebraic invariants are defined for the purpose of gaining insights into solving polynomial equations. Polynomial invariants are disclosed here as an alternative to and to clarify the umbral method of Gian-Carlo Rota. A process for solving cubic polynomial equations is examined and extended to quintic (or 5th degree) polynomial equations.

It is proved that a general cubic polynomial is "apolar" to a quadratic polynomial. It is proved that a quadratic polynomial and cubic polynomial which are apolar either both have repeated roots or both have distinct roots. In the case of repeated roots, these roots are shared by the cubic and quadratic polynomials that are apolar. In the case, in which the derived quadratic which is apolar to a given cubic has distinct roots, it is shown the cubic polynomial $p(x)$ may be transformed to

$$
p(x)=c_{1}\left(x-r_{1}\right)^{3}+c_{2}\left(x-r_{2}\right)^{3},
$$

where $r_{1}$ and $r_{2}$ are the distinct roots of the quadratic polynomial. This will allow the roots of the cubic to be found using algebraic operations.

It will be shown that these methods can be extended to show that a given quintic polynomial is in general apolar to a cubic polynomial. Some remaining questions are posed at the end of the article.
\end{abstract}

AMS Subject Classification: $13 \mathrm{~A} 50$

Key Words: invariant theory, invariants, polynomial equations

Received: July 17, 2013

(c) 2013 Academic Publications, Ltd. url: www.acadpubl.eu 


\section{Introduction}

Gian-Carlo Rota (1932-1999) made contributions to combinatorics with important papers published in the 1960s, and he also made contributions to invariant theory. He expressed the need to bring the theory of invariants back into the forefront of mathematics after being eclipsed by efforts to develop the language of modern algebra during the 20th century.

Invariant Theory is important for gaining knowledge of the intrinsic properties of geometry that transcend coordinate systems. Geometric facts are classically described by means of equations which require a choice of coordinates. Since Descartes, geometric facts have been described using equations in the coordinates $x_{1}, x_{2}, \ldots, x_{n}$. If equations are to express geometric properties, then they must hold no matter what coordinate system is chosen. In other words, equations that describe geometric facts must be invariant under changes of coordinates.

Consider the change of variables given by the translation

$$
x \mapsto x+c \text {, where } c \text { is a constant, }
$$

for a quadratic polynomial $q(x)=x^{2}+2 b_{1} x+b_{2}$, which is written in a special way. If $q(x)$ and $q(x+c)$ each have a double root, then we say this property is invariant under translation.

Let's show this property holds in each case. Since

$$
\begin{aligned}
q(x) & =x^{2}+2 b_{1} x+b_{2} \\
& =x^{2}+2 b_{1} x+b_{1}^{2}+b_{2}-b_{1}^{2} \\
& =\left(x+b_{1}\right)^{2}+b_{2}-b_{1}^{2},
\end{aligned}
$$

$q$ has a double root if and only if $b_{2}-b_{1}^{2}=0$.

Likewise

$$
\begin{aligned}
q(x+c) & =(x+c)^{2}+2 b_{1}(x+c)+b_{2} \\
& =\left(x+c+b_{1}\right)^{2}+b_{2}-b_{1}^{2}
\end{aligned}
$$

has a double root if and only if $b_{2}-b_{1}^{2}=0$.

So the property that a quadratic polynomial has a repeated (or double) root is invariant under translation, and the discriminant $D\left(b_{1}, b_{2}\right)=b_{2}-b_{1}^{2}$ is the desired invariant.

Example. If $q(x)=x^{2}+2(5 x)+17$, then $q(x)=(x+5)^{2}-8$, and with $b_{1}=5$ and $b_{2}=17, D\left(b_{1}, b_{2}\right)=17-5^{2}=-8$. Therefore, $q(x)$ has two distinct roots. 
If $c=4$,

$$
\begin{aligned}
q(x+c) & =(x+c)^{2}+2 \cdot 5(x+c)+17 \\
& =(x+4)^{2}+2 \cdot 5(x+4)+17 \\
& =(x+4+5)^{2}+17-5^{2} \\
& =(x+9)^{2}-8 \\
& =x^{2}+2(9 x)+73 .
\end{aligned}
$$

And we see that $D(73,9)=73-9^{2}=-8$. In either the case, the invariant $D$ evaluated at $q(x)$ or $q(x+4)$ is -8 , and so neither of these quadratic functions has a double root.

Invariant theory is concerned with the problem of finding all invariants of a given set of polynomials, as well as their significance. The significance of an invariant is that every property of polynomials which is invariant under translations is expressed by the vanishing of one or more invariants.

In other words, any set of polynomials which is invariant under translations is the same set as a set of polynomials obtained by setting to zero a set of invariants of such polynomials.

To clarify these statements, we introduce the binomial notation.

\section{Binomial Notation}

Previously we saw a quadratic polynomial of the form

$$
q(x)=x^{2}+2 b_{1} x+b_{2} .
$$

A cubic polyomial written in "binomial" notation has the form

$$
p(x)=a_{0} x^{3}+3 a_{1} x^{2}+3 a_{2} x+a_{3}
$$

so that the coefficients with respect to $x$ include the binomial coefficients $\left(\begin{array}{c}n \\ j\end{array}\right)=\frac{n !}{j !(n-j) !}$, where $n=\operatorname{deg}_{x}(p(x))$, and each term has the form

$$
\left(\begin{array}{c}
n \\
j
\end{array}\right) a_{n-j} x^{j} \text {, whereby } 0 \leq j \leq n .
$$

In this notation, a quintic polynomial will take the form

$$
p(x)=a_{0} x^{5}+5 a_{1} x^{4}+10 a_{2} x^{3}+10 a_{3} x^{2}+5 a_{4} x+a_{5} .
$$

We assume in this paper that all polynomials are in $\mathbf{C}[x]$, where $\mathbf{C}$ is the field of complex numbers. That is, all polynomials have complex coefficients. 


\section{Translation Polynomials}

Let $p(x)$ and $q(x)$ be polynomials in $\mathbf{C}[x]$. Generally we write $p(x)$ and $q(x)$ using the binomial notation:

$$
\begin{gathered}
p(x)=a_{0} x^{n}+\left(\begin{array}{c}
n \\
1
\end{array}\right) a_{1} x^{n-1}+\left(\begin{array}{c}
n \\
2
\end{array}\right) a_{2} x^{n-2}+\cdots+\left(\begin{array}{c}
n \\
n-1
\end{array}\right) a_{n-1} x+a_{n}, \\
q(x)=b_{0} x^{k}+\left(\begin{array}{c}
k \\
1
\end{array}\right) b_{1} x^{k-1}+\left(\begin{array}{c}
k \\
2
\end{array}\right) b_{2} x^{k-2}+\cdots+\left(\begin{array}{c}
k \\
k-1
\end{array}\right) b_{k-1} x+b_{k},
\end{gathered}
$$

where $k=\operatorname{deg}_{x} q(x), n=\operatorname{deg}_{x} p(x)$, and we assume $k \leq n$.

Definition. If $T^{c}$ is the translation operator on a polynomial $p(x)$ defined by $T^{c} p(x)=p(x+c)$, we can write

$$
\begin{aligned}
p(x+c)=p_{0}(c) x^{n}+\left(\begin{array}{c}
n \\
1
\end{array}\right) p_{1}(c) x^{n-1} & +\left(\begin{array}{c}
n \\
2
\end{array}\right) p_{2}(c) x^{n-2} \\
& +\cdots+\left(\begin{array}{c}
n \\
n-1
\end{array}\right) p_{n-1}(c) x+p_{n}(c) .
\end{aligned}
$$

where $p_{j}(c)$, which we refer to as a translation polynomial, is a degree $j$ polynomial in $c$ given by

$$
p_{j}(c)=c^{j} a_{0}+\left(\begin{array}{c}
j \\
1
\end{array}\right) c^{j-1} a_{1}+\left(\begin{array}{c}
j \\
2
\end{array}\right) c^{j-2} a_{2}+\cdots+a_{j}
$$

Example. Consider the cubic polynomial $p(x)=a_{0} x^{3}+3 a_{1} x^{2}+3 a_{2} x+a_{3}$. Then

$$
\begin{aligned}
& T^{c} p(x)=p(x+c)=a_{0}(x+c)^{3}+3 a_{1}(x+c)^{2}+3 a_{2}(x+c)+a_{3} \\
= & a_{0} x^{3}+3\left(a_{0} c+a_{1}\right) x^{2}+3\left(a_{0} c^{2}+2 a_{1} c+a_{2}\right) x+a_{0} c^{3}+3 a_{1} c^{2}+3 a_{2} c+a_{3},
\end{aligned}
$$

so the translation polynomials are $p_{0}(c)=a_{0}, \quad p_{1}(c)=a_{0} c+a_{1}, \quad p_{2}(c)=$ $a_{0} c^{2}+2 a_{1} c+a_{2}$ and $p_{3}(c)=a_{0} c^{3}+3 a_{1} c^{2}+3 a_{2} c+a_{3}$.

Our main focus will be on invariants that depend on the coefficients of two polynomials. 


\section{The Apolar Invariant $A_{2,3}$}

Definitions. A polynomial $I\left(a_{0}, a_{1}, a_{2}, \ldots, a_{n}, b_{0}, b_{1}, b_{2}, \ldots, b_{k}, x\right)$ in the variables $a_{0}, a_{1}, a_{2}, \ldots, a_{n}, b_{0}, b_{1}, b_{2}, \ldots, b_{k}, x$ is said to be an invariant of the two polynomials $p(x)$ and $q(x)$ when

$$
\begin{aligned}
& I\left(a_{0}, a_{1}, a_{2}, \ldots, a_{n}, b_{0}, b_{1}, b_{2}, \ldots, b_{k}, x\right) \\
& \quad=I\left(p_{0}(c), p_{1}(c), p_{2}(c), \ldots, p_{n}(c), q_{0}(c), q_{1}(c), q_{2}(c), \ldots, q_{k}(c), x+c\right),
\end{aligned}
$$

for all complex numbers $c$. Sometimes these invariants are called covariants. For convenience, we use the notation $I(p(x), q(x))$.

Consequently, $I$ is an invariant if and only $I(p(x), q(x))=I\left(T^{c} p(x), T^{c} q(x)\right)$ for all $c$.

Remark. Here we require that $I(p(x), q(x))$ equals $I\left(T^{c} p(x), T^{c} q(x)\right)$ as a polynomial in $n+k+3$ indeterminates. This means that $c$ disappears via cancellation.

Definitions. The apolar invariant $A_{2,3}(q(x), p(x))$ is defined for a quadratic polynomial $q(x)=b_{0} x^{2}+2 b_{1} x+b_{2}$ and cubic polynomial $p(x)=a_{0} x^{3}+3 a_{1} x^{2}+$ $3 a_{2} x+a_{3}$ by

$$
A_{2,3}(q(x), p(x))=\left(a_{0} b_{2}-2 a_{1} b_{1}+b_{0} a_{2}\right) x+2 a_{2} b_{1}-b_{0} a_{3}-a_{1} b_{2} .
$$

We say these two polynomials $p(x)$ and $q(x)$ are $A_{2,3}$-apolar when $A_{2,3}(q(x)$, $p(x))$ is identically 0 , that is, 0 for all $x$.

To verify that $A_{2,3}$ is an invariant, meaning that $A_{2,3}(q(x), p(x))=$ $A_{2,3}\left(T^{c} q(x), T^{c} p(x)\right)$, we can work out the right side of this last equation by simply substituting the translation polynomials $p_{j}(c)$ in for each $a_{j}$ in $A_{2,3}(q(x), p(x))=\left(a_{0} b_{2}-2 a_{1} b_{1}+b_{0} a_{2}\right) x+2 a_{2} b_{1}-b_{0} a_{3}-a_{1} b_{2}$ along with $x+c$ for $x$ and simplify. If $A_{2,3}(q(x), p(x))$ is an invariant, then the $c$ 's will disappear:

$$
\begin{aligned}
A_{2,3}\left(T^{c} q(x), T^{c} p(x)\right)= & A_{2,3}(q(x+c), p(x+c)) \\
= & \left(a_{0}\left(b_{2}+2 b_{1} c+b_{0} c^{2}\right)-2\left(a_{1}+a_{0} c\right)\left(b_{1}+b_{0} c\right)+\right. \\
& \left.+\left(a_{2}+2 a_{1} \cdot c+a_{0} c^{2}\right) b_{0}\right)(x+c) \\
& -\left(a_{1}+a_{0} c\right)\left(b_{2}+2 b_{1} c+b_{0} c^{2}\right) \\
& +2\left(a_{2}+2 a_{1} c+a_{0} c^{2}\right)\left(b_{1}+b_{0} c\right) \\
& -b_{0}\left(a_{3}+3 a_{2} c+3 a_{1} c^{2}+a_{0} c^{3}\right) \\
= & \left(a_{0} b_{2}-2 a_{1} b_{1}+b_{0} a_{2}\right) x+2 a_{2} b_{1}-b_{0} a_{3}-a_{1} b_{2}
\end{aligned}
$$




$$
=A_{2,3}(q(x), p(x)) \text {. }
$$

Therefore, $A_{2,3}(q(x), p(x))$ is an invariant.

Given $p(x)=a_{0} x^{3}+3 a_{1} x^{2}+3 a_{2} x+a_{3}$, a $q(x)=b_{0} x^{2}+2 b_{1} x+b_{2}$ is $A_{2,3}-$ apolar to $p(x)$ if $a_{2} b_{0}-2 a_{1} b_{1}+a_{0} b_{2}=0$ and $a_{3} b_{0}-2 a_{2} b_{1}+a_{1} b_{2}=0$.

So finding $q(x)$ is equivalent to solving a linear system in $b_{0}, b_{1}, b_{2}$ with augmented matrix

$$
\left[\begin{array}{llll}
a_{2} & -2 a_{1} & a_{0} & 0 \\
a_{3} & -2 a_{2} & a_{1} & 0
\end{array}\right]
$$

This will result generally (with some exceptions) in a 1 - dimensional vector space of polynomials $q(x)=b_{0} x^{2}+2 b_{1} x+b_{2}$ that are apolar to the given $p(x)$ as they form the null space of a homogeneous linear system.

Example. Define $p(x)=9 x^{3}-24 x^{2}+11 x+2$.

In binomial form, $p(x)=9 x^{3}+3(-8) x^{2}+3\left(\frac{11}{3}\right) x+2$, so $a_{0}=9, a_{1}=-8$, $a_{2}=\frac{11}{3}, a_{3}=2$.

Solve the system represented by

$$
\left[\begin{array}{cccc}
a_{2} & -2 a_{1} & a_{0} & 0 \\
a_{3} & -2 a_{2} & a_{1} & 0
\end{array}\right]=\left[\begin{array}{cccc}
\frac{11}{3} & -2(-8) & 9 & 0 \\
2 & -2\left(\frac{11}{3}\right) & -8 & 0
\end{array}\right]=\left[\begin{array}{cccc}
\frac{11}{3} & 16 & 9 & 0 \\
2 & -\frac{22}{3} & -8 & 0
\end{array}\right]
$$

which row reduces to $\left[\begin{array}{cccc}1 & 0 & -\frac{279}{265} & 0 \\ 0 & 1 & \frac{213}{265} & 0\end{array}\right]$. So $b_{0}=\frac{279}{265} b_{2}$ and $b_{1}=-\frac{213}{265} b_{2}$.

This gives a 1 - dimensional space of quadratic polynomials apolar to $p(x)$ described by

$$
\begin{aligned}
q(x) & =\frac{279}{265} b_{2}+2\left(-\frac{213}{265} b_{2}\right) x+b_{2} \\
& =b_{2}\left(\frac{279}{265} x^{2}-\frac{426}{265} x+1\right),
\end{aligned}
$$

or alternately by $q(x)=t\left(279 x^{2}-426 x+265\right)$, where $t$ is any complex constant.

Linearity Properties. In a more general setting, the set of quadratic polynomials $S_{p(x)}$ that are found to be $A_{2,3}$ - apolar to a given $p(x)$ emerges as the null space of a homogeneous linear system. Therefore $S_{p(x)}$ is a vector subspace of the vector space $\mathbf{P}_{2}$ of polynomials of degee at most 2 (which itself is isomorphic as a vector space to $\mathbf{C}^{3}$ ). Then $S_{p(x)}$ satisfies the linearity properties of a vector space.

That is: 
1. If $q(x)$ is $A_{2,3}-$ apolar to a given $p(x)$, then so is $c q(x)$ for all constants C.

2. If $q(x)$ and $s(x)$ are each degree at most 2 and apolar to a given cubic $p(x)$, then so is the sum $q(x)+s(x)$. In particular the 0 polynomial is apolar to a given cubic.

If on the other hand we are given a quadratic $q(x)=b_{0} x^{2}+2 b_{1} x+b_{2}$, the task of finding an apolar $p(x)=a_{0} x^{3}+3 a_{1} x^{2}+3 a_{2} x+a_{3}$ which satisfies the equation $A_{2,3}(q(x), p(x))=\left(a_{0} b_{2}-2 a_{1} b_{1}+b_{0} a_{2}\right) x-\left(a_{1} b_{2}-2 a_{2} b_{1}+b_{0} a_{3}\right)=0$, is equivalent to solving a linear system with augmented matrix

$$
\left[\begin{array}{ccccc}
b_{2} & -2 b_{1} & b_{0} & 0 & 0 \\
0 & b_{2} & -2 b_{1} & b_{0} & 0
\end{array}\right]
$$

This in general will produce a 2 - dimensional subspace of polynomials $S_{q(x)}$ of degree at most 3 that are $A_{2,3}$ - apolar to the given quadratic $q(x)$.

This leads to an important property:

Theorem 1. If $q(x)$ is $2 \mathrm{nd}$ degree and $r$ is a root of $q(x)$, then $q(x)$ is apolar to the cubic $f(x)=(x-r)^{3}$.

Proof. This can be shown by expanding $f(x)$ :

$$
f(x)=x^{3}-3 x^{2} r+3 x r^{2}-r^{3} .
$$

In this case, $a_{0}=1, a_{1}=-r, a_{2}=r^{2}$, and $a_{3}=-r^{3}$.

Then

$$
\begin{aligned}
A_{2,3}(q(x), f(x))= & \left(a_{0} b_{2}-2 a_{1} b_{1}+b_{0} a_{2}\right) x \\
& -\left(a_{1} b_{2}-2 a_{2} b_{1}+b_{0} a_{3}\right) \\
= & \left((1) b_{2}-2(-r) b_{1}+b_{0}\left(r^{2}\right)\right) x \\
& -\left((-r) b_{2}-2\left(r^{2}\right) b_{1}+b_{0}\left(-r^{3}\right)\right) \\
= & \left(b_{2}+2 r b_{1}+b_{0} r^{2}\right) x \\
& -\left(b_{2}+2 r b_{1}+b_{0} r^{2}\right)(-r) \\
= & q(r) x-q(r)(-r)=0, \text { since } q(r)=0 .
\end{aligned}
$$

Therefore $q(x)$ is apolar to $(x-r)^{3}$.

If $q(x)$ has roots $r_{1}$ and $r_{2}$, the Linearity Properties (1) and (2) imply that $q(x)$ is $A_{2,3}$ - apolar to $c_{1}\left(x-r_{1}\right)^{3}+c_{2}\left(x-r_{2}\right)^{3}$ for all choices of constants $c_{1}$ and $c_{2}$. In the case in which the roots $r_{1}$ and $r_{2}$ of $q(x)$ are distinct, then 
$\left(x-r_{1}\right)^{3}$ and $\left(x-r_{2}\right)^{3}$ are linearly independent and generate a $2-$ dimensional vector space. Assuming now that $q(x)$ is a quadratic derived initially as apolar to a given cubic $p(x)$, then the the 2 - dimensional vector space of cubics $S_{q(x)}$ that are apolar to $q(x)$ and which contains $p(x)$ equals

$$
S_{q(x)}=\left\{c_{1}\left(x-r_{1}\right)^{3}+c_{2}\left(x-r_{2}\right)^{3} \mid c_{1}, c_{2} \in \mathbf{C}\right\},
$$

so $p(x)=c_{1}\left(x-r_{1}\right)^{3}+c_{2}\left(x-r_{2}\right)^{3}$ for some particular $c_{1}$ and $c_{2}$.

Example. In the previous example, we found that $q(x)=279 x^{2}+2(-213) x+$ 265 is apolar to $p(x)=9 x^{3}-24 x^{2}+11 x+2$.

Solving $279 x^{2}+2(-213) x+265=0 \Rightarrow 279\left(x^{2}+2\left(-\frac{71}{93}\right) x+\frac{265}{279}\right)=0 \Rightarrow$ $\left(x-\frac{71}{93}\right)^{2}+\frac{1058}{2883}=0$, and we find that $x=\frac{71}{93}+\frac{23}{93} i \sqrt{6}$ and $x=\frac{71}{93}-\frac{23}{93} i \sqrt{6}$. So

$$
\begin{aligned}
p(x)=9 x^{3}-24 x^{2}+11 x+2 & \\
= & c_{1}\left(x-\left(\frac{71}{93}+\frac{23}{93} i \sqrt{6}\right)\right)^{3}+c_{2}\left(x-\left(\frac{71}{93}-\frac{23}{93} i \sqrt{6}\right)\right)^{3}
\end{aligned}
$$

for some particular $c_{1}$ and $c_{2}$.

To outline the solution of such a cubic

$$
p(x)=c_{1}\left(x-r_{1}\right)^{3}+c_{2}\left(x-r_{2}\right)^{3}
$$

note that

$$
\begin{aligned}
p(x) & =c_{1}\left(x-r_{1}\right)^{3}+c_{2}\left(x-r_{2}\right)^{3} \\
& =\left(c_{1}+c_{2}\right) x^{3}+\left(-3 c_{1} r_{1}-3 c_{2} r_{2}\right) x^{2}+\left(3 c_{1} r_{1}^{2}+3 c_{2} r_{2}^{2}\right) x-c_{1} r_{1}^{3}-c_{2} r_{2}^{3} \\
& =a_{0} x^{3}+3 a_{1} x^{2}+3 a_{2} x+a_{3},
\end{aligned}
$$

so $c_{1}+c_{2}=a_{0}$ and $-3 c_{1} r_{1}-3 c_{2} r_{2}=3 a_{1}$, which implies that $c_{2}=a_{0}-c_{1}$ and $c_{1} r_{1}+\left(a_{0}-c_{1}\right) r_{2}=-a_{1}$,

$$
\Rightarrow c_{1}=\frac{a_{0} r_{2}+a_{1}}{r_{2}-r_{1}} \text { and } c_{2}=a_{0}-c_{1}=\frac{a_{0} r_{1}+a_{1}}{r_{1}-r_{2}} .
$$

Then $p(x)=\left(\frac{a_{0} r_{2}+a_{1}}{r_{2}-r_{1}}\right)\left(x-r_{1}\right)^{3}+\left(\frac{a_{0} r_{1}+a_{1}}{r_{1}-r_{2}}\right)\left(x-r_{2}\right)^{3}$, so

$$
p(x)=0 \Rightarrow\left(\frac{a_{0} r_{2}+a_{1}}{r_{2}-r_{1}}\right)\left(x-r_{1}\right)^{3}=-\left(\frac{a_{0} r_{1}+a_{1}}{r_{1}-r_{2}}\right)\left(x-r_{2}\right)^{3}
$$




$$
\left(\frac{x-r_{1}}{x-r_{2}}\right)^{3}=\frac{-\left(\frac{a_{0} r_{1}+a_{1}}{r_{1}-r_{2}}\right)}{\left(\frac{a_{0} r_{2}+a_{1}}{r_{2}-r_{1}}\right)}=\frac{a_{0} r_{1}+a_{1}}{a_{0} r_{2}+a_{1}}
$$

Letting $y=\frac{x-r_{1}}{x-r_{2}}$ and $d=\frac{a_{0} r_{1}+a_{1}}{a_{0} r_{2}+a_{1}}$, and using a polar form for $d$, we solve $y^{3}=d$ for the 3 complex roots of $d: y_{0}, y_{1}, y_{2}$ via De Moivre's Theorem.

Use $y_{k}=\frac{x_{k}-r_{1}}{x_{k}-r_{2}}$, to solve for $x_{k}=\frac{y_{k} r_{2}-r_{1}}{y_{k}-1}, k=0,1,2$.

In the example, with $p(x)=9 x^{3}-24 x^{2}+11 x+2$ the above process yields

$$
\begin{aligned}
x_{0}=\frac{y_{0} r_{2}-r_{1}}{y_{0}-1}= & \frac{71}{93}-\frac{212949813507701300000000000000 \sqrt{6}}{578603896932131660122794313233} \\
& -\frac{583686282464563 \sqrt{6}}{578603896932131660122794313233} i \approx-0.138071187458 . \\
x_{1}=\frac{y_{1} r_{2}-r_{1}}{y_{1}-1}= & \frac{15606738876411400000000000000 \sqrt{6}}{925698051533942894964544936329}+\frac{71}{93} \\
& +\frac{248557155553381 \sqrt{6}}{925698051533942894964544936329} i \approx 0.804737854124 .
\end{aligned}
$$

and

$$
\begin{gathered}
x_{2}=\frac{y_{2} r_{2}-r_{1}}{y_{2}-1}=\left(\frac{12115755716993740000000000000 \sqrt{6}}{24000000000008754428964926071}+\frac{71}{93}\right. \\
\left.+\frac{18972058280657 \sqrt{6}}{72000000000026263286894778213} i\right) \approx 2 .
\end{gathered}
$$

Remark: Of course, the above example used to demonstrate this more general process can be solved using the Rational Root Test, division, and quadratic methods.

The previous discussion proves the following.

Corollary 1. Let $p(x)=a_{0} x^{3}+3 a_{1} x^{2}+3 a_{2}+a_{3}$ be a typical cubic and assume the quadratic $q(x)$ is apolar to $p(x)$. If $q(x)$ has two distinct roots $r_{1}$ and $r_{2}$, then

$$
p(x)=\left(\frac{a_{0} r_{2}+a_{1}}{r_{2}-r_{1}}\right)\left(x-r_{1}\right)^{3}+\left(\frac{a_{0} r_{1}+a_{1}}{r_{1}-r_{2}}\right)\left(x-r_{2}\right)^{3} .
$$

Remark. Although $S_{q(x)}$ from the previous example is a vector space that contains cubic polynomials and the zero polynomial, it also contains 2 nd degree polynomials. Consider 


$$
\begin{aligned}
\left(x-\left(\frac{71}{93}+\frac{23}{93} i \sqrt{6}\right)\right)^{3} & -\left(x-\left(\frac{71}{93}-\frac{23}{93} i \sqrt{6}\right)\right)^{3} \\
& =3\left(-\frac{46}{93} i \sqrt{6}\right) x^{2}+3 \cdot \frac{6532}{8649} i \sqrt{6} x-\frac{183218}{268119} i \sqrt{6} .
\end{aligned}
$$

With $g(x)=3\left(-\frac{46}{93} i \sqrt{6}\right) x^{2}+3 \cdot \frac{6532}{8649} i \sqrt{6} x-\frac{183218}{268119} i \sqrt{6}$ in the place of $p(x)$ with $a_{0}=0$, and $q(x)=279 x^{2}+2(-213) x+265$, it checks that $A_{2,3}(q(x)$, $g(x))=0$.

Note. The previous solution of a cubic $p(x)$ depends on the quadratic $q(x)$ that is found to be apolar to the cubic having two distinct roots $r_{1}, r_{2}$. The following proves that if a quadratic has repeated roots, then for an $A_{2,3}-$ apolar $p(x), p(x)=0$ can always be solved by division or similar methods.

Theorem 2. Let quadratic $q(x)=b_{0} x^{2}+2 b_{1} x+b_{2}$ and cubic $p(x)$ be apolar. Then $q(x)$ has a repeated root $x=r$ if and only if $p(x)$ has a repeated root at $x=r$.

Proof. Assuming first that $q(x)=b_{0} x^{2}+2 b_{1} x+b_{2}$ has a repeated root $x=r$, then $q(x)=b_{0}(x-r)^{2}$. Since $b_{0}(x-r)^{2}=b_{0} x^{2}-2 b_{0} r x+b_{0} r^{2}$, then $b_{1}=-b_{0} r$ and $b_{2}=b_{0} r^{2}$. Solving the equation $A_{2,3}(q(x), p(x))=$ $\left(a_{0} b_{2}-2 a_{1} b_{1}+b_{0} a_{2}\right) x-\left(a_{1} b_{2}-2 a_{2} b_{1}+b_{0} a_{3}\right)=0$ for a cubic $p(x)=a_{0} x^{3}+$ $3 a_{1} x^{2}+3 a_{2} x+a_{3}$ with the matrix $\left[\begin{array}{ccccc}b_{2} & -2 b_{1} & b_{0} & 0 & 0 \\ 0 & b_{2} & -2 b_{1} & b_{0} & 0\end{array}\right]$ yields for $q(x)=$ $b_{0} x^{2}-2 b_{0} r x+b_{0} r^{2}$ the augmented matrix $\left[\begin{array}{ccccc}b_{0} r^{2} & 2 b_{0} r & b_{0} & 0 & 0 \\ 0 & b_{0} r^{2} & 2 b_{0} r & b_{0} & 0\end{array}\right]$.

Since $b_{0} \neq 0$, it follows that this system is equivalent to one with the matrix $\left[\begin{array}{ccccc}r^{2} & 2 r & 1 & 0 & 0 \\ 0 & r^{2} & 2 r & 1 & 0\end{array}\right]$ which implies that $r \neq 0$.

Then $\left[\begin{array}{ccccc}r^{2} & 2 r & 1 & 0 & 0 \\ 0 & r^{2} & 2 r & 1 & 0\end{array}\right]$, row reduces to $\left[\begin{array}{ccccc}1 & 0 & -\frac{3}{r^{2}} & -\frac{2}{r^{3}} & 0 \\ 0 & 1 & \frac{2}{r} & \frac{1}{r^{2}} & 0\end{array}\right]$.

So $a_{0}=a_{2} \frac{3}{r^{2}}+a_{3} \frac{2}{r^{3}}=\frac{3 r a_{2}+2 a_{3}}{r^{3}}$ and $a_{1}=-a_{2} \frac{2}{r}-a_{3} \frac{1}{r^{2}}=\frac{-2 r a_{2}-a_{3}}{r^{2}}$.

Therefore, a cubic $p(x)$ that is apolar to $q(x)=b_{0}(x-r)^{2}$ must have the form

$$
p(x)=\left(\frac{3 r a_{2}+2 a_{3}}{r^{3}}\right) x^{3}+3\left(\frac{-2 r a_{2}-a_{3}}{r^{2}}\right) x^{2}+3 a_{2} x+a_{3} .
$$

Since

$$
\begin{array}{r}
\left(\frac{\left(3 a_{2} r+2 a_{3}\right) x+r a_{3}}{r^{3}}\right)(x-r)^{2}=\left(\frac{3 r a_{2}+2 a_{3}}{r^{3}}\right) x^{3}+3\left(\frac{-2 r a_{2}-a_{3}}{r^{2}}\right) x^{2} \\
+3 a_{2} x+a_{3}
\end{array}
$$


then

$$
p(x)=\left(\frac{\left(3 a_{2} r+2 a_{3}\right) x+r a_{3}}{r^{3}}\right)(x-r)^{2},
$$

so $p(x)$ has the repeated root $r$.

On the other hand, if $p(x)$ has a repeated root $x=r$, then

$p(x)=a_{0}(x-r)^{2}(x-a)=a_{0} x^{3}+\left(-2 a_{0} r-a_{0} a\right) x^{2}+\left(a_{0} r^{2}+2 a_{0} r a\right) x-a_{0} r^{2} a$.

So then $a_{3}=-a_{0} r^{2} a, a_{2}=\frac{a_{0}\left(r^{2}+2 r a\right)}{3}$, and $a_{1}=-\frac{a_{0}(2 r+a)}{3}$.

The system with with augmented matrix $\left[\begin{array}{llll}a_{2} & -2 a_{1} & a_{0} & 0 \\ a_{3} & -2 a_{2} & a_{1} & 0\end{array}\right]$, which is equivalent to $\left[\begin{array}{llll}a_{0} & -2 a_{1} & a_{2} & 0 \\ a_{1} & -2 a_{2} & a_{3} & 0\end{array}\right]$ becomes, with the above substitutions,

$$
\begin{gathered}
{\left[\begin{array}{cccc}
a_{0} & 2 \frac{a_{0}(2 r+a)}{3} & \frac{a_{0}\left(r^{2}+2 r a\right)}{3} & 0 \\
-\frac{a_{0}(2 r+a)}{3} & -2 \frac{a_{0}\left(r^{2}+2 r a\right)}{3} & -a_{0} r^{2} a & 0
\end{array}\right] \text {, which row reduces to }} \\
\\
{\left[\begin{array}{cccc}
1 & 0 & -r^{2} & 0 \\
0 & 1 & r & 0
\end{array}\right] .}
\end{gathered}
$$

This means that $b_{2}=r^{2} b_{0}$ and $b_{1}=-r b_{0}$, so that

$$
q(x)=b_{0} x^{2}-2 r b_{0} x+r^{2} b_{0}=b_{0}(x-r)^{2}
$$

is the typical quadratic polynomial apolar to a given cubic polynomial with repeated roots.

Note. Before leaving our discussion of the $A_{2,3}$ invariant, it is worth noting that not every cubic polynomial is $A_{2,3}-$ apolar to a nonzero quadratic polynomial. Consider $p(x)=x^{3}+3 x^{2}+3 x+2$. In this case, $a_{0}=1, a_{1}=1$, $a_{2}=1$, and $a_{3}=2$. In solving the linear system represented by

$$
\left[\begin{array}{llll}
a_{2} & -2 a_{1} & a_{0} & 0 \\
a_{3} & -2 a_{2} & a_{1} & 0
\end{array}\right]=\left[\begin{array}{cccc}
1 & -2 & 1 & 0 \\
2 & -2 & 1 & 0
\end{array}\right]
$$

which row reduces to $\left[\begin{array}{cccc}1 & 0 & 0 & 0 \\ 0 & 1 & -\frac{1}{2} & 0\end{array}\right]$, we get that $b_{0}=0$, and $b_{1}=\frac{1}{2} b_{2}$, and we have that $q(x)=2\left(\frac{1}{2} b_{2}\right) x+b_{2}=b_{2}(x+1)$ is apolar to $p(x)=x^{3}+3 x^{2}+$ $3 x+2$ for every choice of $b_{2}$. 


\section{The General Apolar Invariant $A_{k, n}$}

Definitions. For integers $k$ and $n$ with $1 \leq k \leq n$, define the polynomials

$$
\begin{aligned}
T_{i}=(-1)^{i}\left(\begin{array}{c}
n-k \\
i
\end{array}\right) x^{n-k-i}\left(a_{i} b_{k}-\left(\begin{array}{c}
k \\
1
\end{array}\right) a_{i+1} b_{k-1}+\cdots\right. \\
\left.\cdots+(-1)^{k-1}\left(\begin{array}{c}
k \\
k-1
\end{array}\right) a_{k+i-1} b_{1}+(-1)^{k} a_{k+i} b_{0}\right)
\end{aligned}
$$

for $0 \leq i \leq n-k$.

The general apolar expression $A_{k, n}$ is defined by

$$
A_{k, n}(q(x), p(x))=\sum_{i=0}^{n-k} T_{i} .
$$

If $A_{k, n}(q(x+c), p(x+c))=A_{k, n}(q(x), p(x))$ for all $c$, then $A_{k, n}$ is an apolar invariant.

If $A_{k, n}(q(x), p(x))=0$ for all $x$, we will say that $q(x)$ and $p(x)$ are $A_{k, n}-$ apolar.

Example. We know from before that for $q(x)=b_{0} x^{2}+2 b_{1} x+b_{0}$ and $p(x)=a_{0} x^{3}+3 a_{1} x^{2}+3 a_{2} x+a_{3}$,

$$
A_{2,3}(q(x), p(x))=x\left(a_{0} b_{2}-2 a_{1} b_{1}+a_{2} b_{0}\right)-\left(a_{1} b_{2}-2 a_{2} b_{1}+a_{3} b_{0}\right)
$$

is an invariant, and this agrees with the above general formula when $k=2$ and $n=3$.

The following generalizes Theorem 1.

Theorem 3. Let $q(x)$ be a polynomial of degree $k$, and let $n$ be an integer with $n \geq k$. If $A_{k, n}$ is an invariant, and $r$ is a root of $q(x)$, then $q(x)$ is $A_{k, n}-$ apolar to $(x-r)^{n}$.

Proof. Let $p(x)=(x-r)^{n}$.

$$
\begin{aligned}
p(x)= & x^{n}+n(-r) x^{n-1}+\left(\begin{array}{c}
n \\
2
\end{array}\right)(-r)^{2} x^{n-2}+\cdots \\
& \cdots+\left(\begin{array}{c}
n \\
n-2
\end{array}\right)(-r)^{n-2} x^{2}+\left(\begin{array}{c}
n \\
n-1
\end{array}\right)(-r)^{n-1} x+(-r)^{n}
\end{aligned}
$$

so that for

$$
p(x)=a_{0} x^{n}+\left(\begin{array}{c}
n \\
1
\end{array}\right) a_{1} x^{n-1}+\left(\begin{array}{c}
n \\
2
\end{array}\right) a_{2} x^{n-2}+\cdots+\left(\begin{array}{c}
n \\
n-1
\end{array}\right) a_{n-1} x+a_{n},
$$


we have

$$
a_{0}=1, a_{1}=-r, a_{2}=(-r)^{2}, \text { and } a_{j}=(-r)^{j}
$$

for all $j \in[0, n] \cap \mathbf{Z}$.

We need to show that $A_{k, n}(q(x), p(x))=0$. For this, it's sufficient to show for each $i$ with $0 \leq i \leq n-k$ that

$$
\begin{gathered}
\left(a_{i} b_{k}-\left(\begin{array}{c}
k \\
1
\end{array}\right) a_{i+1} b_{k-1}+\cdots+(-1)^{k-1}\left(\begin{array}{c}
k \\
k-1
\end{array}\right) a_{k+i-1} b_{1}+(-1)^{k} a_{k+i} b_{0}\right) \\
=0
\end{gathered}
$$

This will prove that each term $T_{i}$ of $A_{k, n}(q(x), p(x))$ is 0 .

Considering (1), we have

$$
\begin{aligned}
& \left(a_{i} b_{k}-\left(\begin{array}{c}
k \\
1
\end{array}\right) a_{i+1} b_{k-1}+\cdots+(-1)^{k-1}\left(\begin{array}{c}
k \\
k-1
\end{array}\right) a_{k+i-1} b_{1}\right. \\
& \left.+(-1)^{k} a_{k+i} b_{0}\right) \\
& =\left((-r)^{i} b_{k}-\left(\begin{array}{c}
k \\
1
\end{array}\right)(-r)^{i+1} b_{k-1}+\cdots+(-1)^{k-1}\left(\begin{array}{c}
k \\
k-1
\end{array}\right)(-r)^{k+i-1} b_{1}\right. \\
& \left.+(-1)^{k}(-r)^{k+i} b_{0}\right) \\
& =(-r)^{i}\left(b_{k}-\left(\begin{array}{c}
k \\
1
\end{array}\right)(-r)^{1} b_{k-1}+\cdots+(-1)^{k-1}\left(\begin{array}{c}
k \\
k-1
\end{array}\right)(-r)^{k-1} b_{1}\right. \\
& \left.+(-1)^{k}(-r)^{k} b_{0}\right) \\
& =(-r)^{i}\left(b_{k}+\left(\begin{array}{c}
k \\
1
\end{array}\right) r b_{k-1}+\cdots+\left(\begin{array}{c}
k \\
k-1
\end{array}\right) r^{k-1} b_{1}+r^{k} b_{0}\right) \\
& =(-r)^{i} q(r)=0 \text { since } q(r)=0 \text {. }
\end{aligned}
$$

Therefore, $A_{k, n}(q(x), p(x))=0$, and $q(x)$ is $A_{k, n}-$ apolar to $(x-r)^{n}$.

Theorem. (Rota) For positive integers $k$ and $n$ with $k \leq n$ :

(a) The dimension of the space of all polynomials of degree $k$ which are apolar to a polynomial of degree $n$ (with $n<2 k$ ) equals $2 k-n$, in general.

(b) The dimension of the space of all polynomials of degree $n$ which are apolar to a polynomial of degree $k$ equals $k$, see [2].

Example. The apolar invariant $A_{3,5}$. 
Definitions. The apolar invariant $A_{3,5}(q(x), p(x))$ is defined for a cubic polynomial $q(x)=b_{0} x^{3}+3 b_{1} x^{2}+3 b_{2} x+b_{3}$ and quintic polynomial $p(x)=$ $a_{0} x^{5}+5 a_{1} x^{4}+10 a_{2} x^{3}+10 a_{3} x^{2}+5 a_{4} x+a_{5}$ as

$$
\begin{aligned}
& A_{3,5}(q(x), p(x))=x^{2}\left(a_{0} b_{3}-3 a_{1} b_{2}+3 a_{2} b_{1}-a_{3} b_{0}\right) \\
& -2 x\left(a_{1} b_{3}-3 a_{2} b_{2}+3 a_{3} b_{1}-a_{4} b_{0}\right)+a_{2} b_{3}-3 a_{3} b_{2}+3 a_{4} b_{1}-a_{5} b_{0} .
\end{aligned}
$$

We say these polynomials $p(x)$ and $q(x)$ are $A_{3,5}$ - apolar when $A_{3,5}(q(x), p(x))$ is 0 for all $x$.

To prove that $A_{3,5}(q(x), p(x))$ is an invariant, that is, $A_{3,5}(q(x), p(x))=$ $A_{3,5}\left(T^{c} q(x), T^{c} p(x)\right)$ for all $c$, note that for

$$
\begin{aligned}
A_{3,5}(q(x), p(x))= & x^{2}\left(a_{0} b_{3}-3 a_{1} b_{2}+3 a_{2} b_{1}\right. \\
& \left.-a_{3} b_{0}\right)-2 x\left(a_{1} b_{3}-3 a_{2} b_{2}+3 a_{3} b_{1}-a_{4} b_{0}\right) \\
& +a_{2} b_{3}-3 a_{3} b_{2}+3 a_{4} b_{1}-a_{5} b_{0},
\end{aligned}
$$

$$
\begin{aligned}
A_{3,5}\left(T^{c} q(x), T^{c} p(x)\right)= & (x+c)^{2}\left(a_{0}\left(b_{3}+3 b_{2} c+3 b_{1} c^{2}+b_{0} c^{3}\right)\right. \\
& -3\left(a_{1}+a_{0} c\right)\left(b_{2}+2 b_{1} c+b_{0} c^{2}\right) \\
& +\cdots+3\left(a_{2}+2 a_{1} c+a_{0} c^{2}\right)\left(b_{1}+b_{0} c\right) \\
& \left.-\left(a_{3}+3 a_{2} c+3 a_{1} c^{2}+a_{0} c^{3}\right) b_{0}\right) \\
& -2(x+c)\left(\left(a_{1}+a_{0} c\right)\left(b_{3}+3 b_{2} c+3 b_{1} c^{2}+b_{0} c^{3}\right)\right. \\
& -3\left(a_{2}+2 a_{1} c+a_{0} c^{2}\right)\left(b_{2}+2 b_{1} c+b_{0} c^{2}\right) \\
& +\cdots+3\left(a_{3}+3 a_{2} c+3 a_{1} c^{2}+a_{0} c^{3}\right)\left(b_{1}+b_{0} c\right) \\
& \left.-\left(a_{4}+4 a_{3} c+6 a_{2} c^{2}+4 a_{1} c^{3}+a_{0} c^{4}\right) b_{0}\right) \\
& +\left(\left(a_{2}+2 a_{1} c+a_{0} c^{2}\right)\left(b_{3}+3 b_{2} c+3 b_{1} c^{2}+b_{0} c^{3}\right)\right. \\
& -\cdots-3\left(a_{3}+3 a_{2} c+3 a_{1} c^{2}+a_{0} c^{3}\right)\left(b_{2}+2 b_{1} c+b_{0} c^{2}\right) \\
& +\cdots+3\left(a_{4}+4 a_{3} c+6 a_{2} c^{2}+4 a_{1} c^{3}+a_{0} c^{4}\right)\left(b_{1}+b_{0} c\right) \\
& \left.-\cdots-\left(a_{5}+5 a_{4} \cdot c+10 a_{3} c^{2}+10 a_{2} c^{3}+5 a_{1} c^{4}+a_{0} c^{5}\right) b_{0}\right) \\
= & x^{2}\left(a_{0} b_{3}-3 a_{1} b_{2}+3 a_{2} b_{1}-a_{3} b_{0}\right) \\
& -2 x\left(a_{1} b_{3}-3 a_{2} b_{2}+3 a_{3} b_{1}-a_{4} b_{0}\right) \\
& +a_{2} b_{3}-3 a_{3} b_{2}+3 a_{4} b_{1}-a_{5} b_{0} \\
= & A_{3,5}(q(x), p(x)) .
\end{aligned}
$$

Therefore, $A_{3,5}$ is an invariant to which Th. 3 applies. 
By this new definition of apolarity, given a degree 5 polynomial $p(x)=$ $a_{0} x^{5}+5 a_{1} x^{4}+10 a_{2} x^{3}+10 a_{3} x^{2}+5 a_{4} x+a_{5}$, the coefficients of an apolar cubic polynomial $q(x)=b_{0} x^{3}+3 b_{1} x^{2}+3 b_{2} x+b_{3}$ would need to satisfy the equations in the 4 variables $b_{0}, b_{1}, b_{2}, b_{3}$ :

$$
\begin{aligned}
& a_{3} b_{0}-3 a_{2} b_{1}+3 a_{1} b_{2}-a_{0} b_{3}=0 \\
& a_{4} b_{0}-3 a_{3} b_{1}+3 a_{2} b_{2}-a_{1} b_{3}=0, \\
& a_{5} b_{0}-3 a_{4} b_{1}+3 a_{3} b_{2}-a_{2} b_{3}=0 .
\end{aligned}
$$

This will generally produce a 1 - dimensional vector space of cubic polynomials each apolar to the given quintic, which agrees with Rota's Theorem. Alternately, given a cubic polynomial $q(x)=b_{0} x^{3}+3 b_{1} x^{2}+3 b_{2} x+b_{3}$, the $A_{3,5}-$ invariant definition likewise gives a homogenous linear system in 6 variables a quintic $p(x)$ would need to satisfy:

$$
\begin{aligned}
a_{0} b_{3}-3 a_{1} b_{2}+3 a_{2} b_{1}-a_{3} b_{0} & =0 \\
a_{1} b_{3}-3 a_{2} b_{2}+3 a_{3} b_{1}-a_{4} b_{0} & =0 \\
a_{2} b_{3}-3 a_{3} b_{2}+3 a_{4} b_{1}-a_{5} b_{0} & =0 .
\end{aligned}
$$

which in general will result in a $3-$ dimensional vector space of quintic polynomials each apolar to the given cubic.

Given a quintic $p(x)=a_{0} x^{5}+5 a_{1} x^{4}+10 a_{2} x^{3}+10 a_{3} x^{2}+5 a_{4} x+a_{5}$ Th. 3 implies that if $r$ is the root of an $A_{3,5}-$ apolar cubic polynomial $q(x)$, then $q(x)$ is $A_{3,5}-$ apolar to $(x-r)^{5}$. Then if $r_{1}, r_{2}, r_{3}$ are the distinct roots of $q(x)$, linearity properties imply that $p(x)=c_{1}\left(x-r_{1}\right)^{5}+c_{2}\left(x-r_{2}\right)^{5}+c_{3}\left(x-r_{3}\right)^{5}$ for particular constants $c_{1}, c_{2}, c_{3}$.

For the quintic, $p(x)=2 x^{5}+7 x^{4}-36 x^{3}-107 x^{2}+14 x+120$, we need only consider using the monic associate of $p(x)$,

$$
\frac{1}{2} \cdot p(x)=x^{5}+\frac{7}{2} x^{4}-18 x^{3}-\frac{107}{2} x^{2}+7 x+60
$$

in the calculations. So, let's have a couple of definitions before delving into this example.

Definitions. An associate of a polynomial $p(x)$ with coefficients in a field, such as the complex numbers, is any nonzero multiple of $p(x)$. That is, $c p(x)$ is an associate of $p(x)$. if $c \neq 0$. A monic polynomial is a polynomial with leading coefficient of 1 , so a monic associate of a polynomial $p(x)$ equals $\frac{1}{a_{0}} \cdot p(x)$, if $a_{0}$ is the leading coefficient of $p(x)$. 
Note. Due to the linearity properties discussed previously, we can find the space of cubic polynomials that are apolar to $p(x)=2 x^{5}+7 x^{4}-36 x^{3}-$ $107 x^{2}+14 x+120$ by finding the space of cubic polynomials apolar to the monic associate of $p(x): \frac{1}{2} \cdot p(x)=x^{5}+\frac{7}{2} x^{4}-18 x^{3}-\frac{107}{2} x^{2}+7 x+60$.

Further it's sufficient to seek only the monic cubic polynomials that are apolar to $f(x)=x^{5}+\frac{7}{2} x^{4}-18 x^{3}-\frac{107}{2} x^{2}+7 x+60$. This will simplify the system of equations

$$
\begin{aligned}
& 3 a_{2} b_{1}-3 a_{1} b_{2}+a_{0} b_{3}=a_{3} b_{0}, \\
& 3 a_{3} b_{1}-3 a_{2} b_{2}+a_{1} b_{3}=a_{4} b_{0}, \\
& 3 a_{4} b_{1}-3 a_{3} b_{2}+a_{2} b_{3}=a_{5} b_{0},
\end{aligned}
$$

that needs to be solved to the nonhomogeneous system

$$
\begin{aligned}
3 a_{2} b_{1}-3 a_{1} b_{2}+b_{3} & =a_{3}, \\
3 a_{3} b_{1}-3 a_{2} b_{2}+a_{1} b_{3} & =a_{4}, \\
3 a_{4} b_{1}-3 a_{3} b_{2}+a_{2} b_{3} & =a_{5},
\end{aligned}
$$

that has the augmented matrix

$$
\left[\begin{array}{cccc}
3 a_{2} & -3 a_{1} & 1 & a_{3} \\
3 a_{3} & -3 a_{2} & a_{1} & a_{4} \\
3 a_{4} & -3 a_{3} & a_{2} & a_{5}
\end{array}\right]
$$

For

$$
\begin{aligned}
f(x)= & x^{5}+\frac{7}{2} x^{4}-18 x^{3}-\frac{107}{2} x^{2}+7 x+60 \\
& =x^{5}+5\left(\frac{7}{10}\right) x^{4}+10\left(-\frac{9}{5}\right) x^{3}+10\left(-\frac{107}{20}\right) x^{2}+5\left(\frac{7}{5}\right) x+60 \\
& a_{1}=\frac{7}{10}, \quad a_{2}=-\frac{9}{5}, \quad a_{3}=-\frac{107}{20}, \quad a_{4}=\frac{7}{5}, \text { and } a_{5}=60 .
\end{aligned}
$$

The matrix

$$
\left[\begin{array}{cccc}
3\left(-\frac{9}{5}\right) & -3\left(\frac{7}{10}\right) & 1 & -\frac{107}{20} \\
3\left(-\frac{107}{20}\right) & -3\left(-\frac{9}{5}\right) & \frac{7}{10} & \frac{7}{5} \\
3\left(\frac{7}{5}\right) & -3\left(-\frac{107}{20}\right) & -\frac{9}{5} & 60
\end{array}\right]=\left[\begin{array}{cccc}
-\frac{27}{5} & -\frac{21}{10} & 1 & -\frac{107}{20} \\
-\frac{321}{20} & \frac{27}{5} & \frac{7}{10} & \frac{7}{5} \\
\frac{21}{5} & \frac{321}{20} & -\frac{9}{5} & 60
\end{array}\right]
$$

row reduces to

$$
\left[\begin{array}{llll}
1 & 0 & 0 & \frac{4657}{1854} \\
0 & 1 & 0 & \frac{4853}{927} \\
0 & 0 & 1 & \frac{23741}{1236}
\end{array}\right]
$$


So $b_{1}=\frac{4657}{1854}, b_{2}=\frac{4853}{927}, b_{3}=\frac{23741}{1236}$, and

$$
q(x)=x^{3}+3\left(\frac{4657}{1854}\right) x^{2}+3\left(\frac{4853}{927}\right) x+\frac{23741}{1236}
$$

is apolar to $p(x)=2 x^{5}+7 x^{4}-36 x^{3}-107 x^{2}+14 x+120$.

To find a quadratic $g(x)=d_{0} x^{2}+2 d_{1} x+d_{2}$ apolar to this $q(x)$, we solve the system of equations with augmented matrix

$$
\left[\begin{array}{cccc}
b_{2} & -2 b_{1} & b_{0} & 0 \\
b_{3} & -2 b_{2} & b_{1} & 0
\end{array}\right]=\left[\begin{array}{cccc}
\frac{4853}{927} & -2\left(\frac{4657}{1854}\right) & 1 & 0 \\
\frac{23741}{1236} & -2\left(\frac{4853}{927}\right) & \left(\frac{4657}{1854}\right) & 0
\end{array}\right],
$$

which row reduces to $\left[\begin{array}{cccc}1 & 0 & \frac{7385450}{143272639} & 0 \\ 0 & 1 & -\frac{20822879}{143272639} & 0\end{array}\right]$.

This gives

$$
d_{0}=-\frac{7385450}{143272639} d_{2}, \text { and } d_{1}=\frac{20822879}{143272639} d_{2},
$$

and

$$
g(x)=-7385450 x^{2}+2(20822879) x+143272639
$$

is a convenient associate.

Solving $g(x)=0$ yields the equation

$$
\begin{aligned}
& x^{2}-2\left(\frac{20822879}{7385450}\right) x-\frac{143272639}{7385450}=0 \\
\Rightarrow & \left(x-\frac{20822879}{7385450}\right)^{2}-\frac{1491725201551191}{54544871702500}=0 \\
\Rightarrow & x=\frac{20822879}{7385450} \pm \sqrt{\frac{1491725201551191}{54544871702500}} .
\end{aligned}
$$

With $g(x)$ having 2 distinct roots, Th. 2 implies that the cubic $q(x)$ has 3 distinct roots $r_{1}, r_{2}$, and $r_{3}$ as well, which may be found using Cor. 1 . Therefore, Th. 3 and linearity properties imply that

$$
p(x)=k_{1}\left(x-r_{1}\right)^{5}+k_{2}\left(x-r_{2}\right)^{5}+k_{3}\left(x-r_{3}\right)^{5}
$$

for particular constants $k_{1}, k_{2}, k_{3}$.

Example. The apolar invariant $A_{2,5}$ For a quadratic $q(x)=b_{0} x^{2}+2 b_{1} x+b_{2}$ and quintic $p(x)=a_{0} x^{5}+5 a_{1} x^{4}+10 a_{2} x^{3}+10 a_{3} x^{2}+5 a_{4} x+a_{5}$, the apolar expression

$$
A_{2,5}(q(x), p(x))=\left(b_{2} a_{3}-2 b_{1} a_{4}+b_{0} a_{5}\right)-3 x\left(b_{2} a_{2}-2 b_{1} a_{3}+b_{0} a_{4}\right)
$$




$$
+3 x^{2}\left(b_{2} a_{1}-2 b_{1} a_{2}+b_{0} a_{3}\right)-x^{3}\left(b_{2} a_{0}-2 b_{1} a_{1}+b_{0} a_{2}\right),
$$

can be proved to be an invariant similar to the invariants $A_{2,3}$ and $A_{3,5}$. However the task of finding a quadratic $q(x)$ apolar to a given quintic $p(x)$ involves solving the system

$$
\begin{aligned}
& b_{2} a_{3}-2 b_{1} a_{4}+b_{0} a_{5}=0, \\
& b_{2} a_{2}-2 b_{1} a_{3}+b_{0} a_{4}=0, \\
& b_{2} a_{1}-2 b_{1} a_{2}+b_{0} a_{3}=0 \\
& b_{2} a_{0}-2 b_{1} a_{1}+b_{0} a_{2}=0
\end{aligned}
$$

which will generally yield a nonzero $q(x)$ only in special cases. This agrees with Rota's Theorem, which requires that $n<2 k$, which does not follow when $n=5$ and $k=2$.

A nonzero solution to this overdetermined system, which can be solved by use of the augmented matrix

$$
\left[\begin{array}{llll}
a_{3} & -2 a_{4} & a_{5} & 0 \\
a_{2} & -2 a_{3} & a_{4} & 0 \\
a_{1} & -2 a_{2} & a_{3} & 0 \\
a_{0} & -2 a_{1} & a_{2} & 0
\end{array}\right]
$$

which reduces to

$$
\left[\begin{array}{cccc}
a_{3} & -2 a_{4} & a_{5} & 0 \\
0 & -2 a_{3}^{2}+2 a_{2} a_{4} & a_{4} a_{3}-a_{2} a_{5} & 0 \\
0 & 0 & -2 a_{3}^{3}+4 a_{3} a_{2} a_{4}+2 a_{1} a_{5} a_{3}-2 a_{2}^{2} a_{5}-2 a_{1} a_{4}^{2} & 0 \\
0 & 0 & 0 & 0
\end{array}\right]
$$

only occurs if

$$
-2 a_{3}^{3}+4 a_{3} a_{2} a_{4}+2 a_{1} a_{5} a_{3}-2 a_{2}^{2} a_{5}-2 a_{1} a_{4}^{2}=0,
$$

which is true only in special cases.

Example. For

$$
\begin{gathered}
p(x)=2 x^{5}-5 x^{4}+130 x^{3}-190 x^{2}+485 x-211, \\
p(x)=2 x^{5}+5(-1) x^{4}+10(13) x^{3}+10(-19) x^{2}+5(97) x-211,
\end{gathered}
$$

so $a_{0}=2, a_{1}=-1, a_{2}=13, a_{3}=-19, a_{4}=97, a_{5}=-211$. 
The polynomial

$$
-2 a_{3}^{3}+4 a_{3} a_{2} a_{4}+2 a_{1} a_{5} a_{3}-2 a_{2}^{2} a_{5}-2 a_{1} a_{4}^{2}
$$

takes on the value

$$
\begin{aligned}
-2(-19)^{3}+4(-19)(13)(97)+2(-1) & (-211)(-19) \\
& =-2(13)^{2}(-211)-2(-1)(97)^{2}=0 .
\end{aligned}
$$

So,

$$
\left[\begin{array}{llll}
a_{3} & -2 a_{4} & a_{5} & 0 \\
a_{2} & -2 a_{3} & a_{4} & 0 \\
a_{1} & -2 a_{2} & a_{3} & 0 \\
a_{0} & -2 a_{1} & a_{2} & 0
\end{array}\right]=\left[\begin{array}{cccc}
-19 & -2(97) & -211 & 0 \\
13 & -2(-19) & 97 & 0 \\
-1 & -2(13) & -19 & 0 \\
2 & -2(-1) & 13 & 0
\end{array}\right]
$$

which reduces to

$$
\left[\begin{array}{llll}
1 & 0 & 6 & 0 \\
0 & 1 & \frac{1}{2} & 0 \\
0 & 0 & 0 & 0 \\
0 & 0 & 0 & 0
\end{array}\right]
$$

gives that $b_{2}=-6 b_{0}$ and $b_{1}=-\frac{1}{2} b_{0}$.

Therefore $q(x)=b_{0} x^{2}+2 b_{1} x+b_{2}=b_{0}\left(x^{2}-x-6\right)$ is $A_{2,5}-$ apolar to $p(x)$ for all $b_{0}$. Previous methods springing from truths governing vector spaces apply to produce that $p(x)=(x+2)^{5}+(x-3)^{5}$, which can be solved by radicals, in this special case.

\section{Questions}

1. Theorem 2 implies that a quadratic polynomial has repeated roots if and only if an apolar cubic polynomial also has repeated roots. Can a similar connection be found between an apolar cubic and quintic?

2. In light of Question 1, given that the discriminant of a polynomial provides information regarding whether the roots of the polynomial are distinct or not, what connection(s) can be found between an apolar invariant and the discriminant of a polynomial?

3. What connections might be found between apolar invariant theory and what Galois Theory tells us regarding the solvability of a 5th-degree polynomial equation? 
4. Since the form generally available for an arbitrary quintic $p(x)$ is $p(x)=$ $k_{1}\left(x-r_{1}\right)^{5}+k_{2}\left(x-r_{2}\right)^{5}+k_{3}\left(x-r_{3}\right)^{5}$, what methods might there be for solving a quintic polynomial equation of this form $k_{1}\left(x-r_{1}\right)^{5}+k_{2}\left(x-r_{2}\right)^{5}+$ $k_{3}\left(x-r_{3}\right)^{5}=0$ ?

5. Given that special cases of the general apolar expression $A_{k, n}$ have been proven to be invariants, can a general rigorous proof be found to show that $A_{k, n}$ is an invariant for all positive integers $k$ and $n$ ?

\section{References}

[1] Joseph P.S. Kung, Gian-Carlo Rota, The invariant theory of binary forms, Bulletin (New Series) of the American Mathematical Society, 10, No. 1 (1984).

[2] Gian-Carlo Rota, Invariant theory, old and new, In: Colloquium Lecture delivered at the Annual Meeting of the American Mathematical Society and Mathematical Association of America, Baltimore MD, January 8, 1998. 